\title{
Vascular complications in two kidney allograft recipients secondary to different fungal infections from the same donor: A case report.
}

\author{
Gongbin Lan ${ }^{1}$, Decheng Deng ${ }^{2}$, Xubiao Xie ${ }^{1}$, Chunhua Fang ${ }^{1}$, Lei Liu ${ }^{1}$, Helong Dai ${ }^{1}$, Longkai Peng1 ${ }^{*}$ \\ ${ }^{1}$ Center of Organ Transplantation, the Second Xiangya Hospital of Central South University, Changsha 410011, PR \\ China \\ ${ }^{2}$ Department of Kidney Transplantation, Zhongshan Hospital of Sun Yat-sen University, Zhongshan 528403, PR China
}

\begin{abstract}
Donor-derived fungal infection is a significant complication in renal transplant recipients that can be associated with serious and sometimes deadly consequences. This article reports two patients who develop severe vascular complications secondary to different fungal infections from the same donor. One patient is diagnosed with renal artery aneurysm that is caused by Candida parapsilosis, and the graft has to be removed. The other patient has developed renal arterial rupture that is attributed to Cryptococcus. Although the affected renal artery is excised and the remaining artery is anastomosed, the function of the graft is not restored. Early recognition and prompt management of vascular complications caused by fungal infections are crucial for favorable outcomes.
\end{abstract}

Keywords: Renal transplantation, Aneurysm, Donor-derived infection.

Accepted on October 30, 2017

\section{Introduction}

Renal artery aneurysm or rupture caused by mycotic infection is an extremely rare but potentially life-threatening complication after renal transplantation [1,2], with an estimated prevalence of less than $0.1 \%$ [3]. The infection can be derived from the donor, exogenous contamination during harvesting, preservation and implantation of the graft $[4,5]$, or preexistent infection in the recipient [6]. Early recognition and prompt management of renal artery complication is very important because of its devastating clinical course and outcomes. Herein we report two recipients who have underwent renal transplantation from the same donor and developed renal artery aneurysm or rupture secondary to different mycotic infections during early post-transplant period.

\section{Case Report}

\section{Donor}

A 38-year-old man died of brain injury secondary to brain and abdominal trauma. Five days after being admitted to the intensive care unit, the patient was diagnosed with brain death and accepted as a multi-organ donor. The usual blood and urine culture were negative, although the patient had pulmonary infection and carried a urinary catheter before organ extraction. The serum creatinine level of the donor was $1.2 \mathrm{mg} / \mathrm{dL}$. Results of hepatitis $\mathrm{B}$ and $\mathrm{C}$ viruses were negative, and human immunodeficiency virus test result was also negative. The liver and kidneys were removed and allocated to different transplant centers.

\section{Renal recipient one}

Patient 1 was a 62-year-old woman with end-stage renal disease caused by polycystic kidney. Three years after starting hemodialysis, she underwent deceased-donor kidney transplantation of the right kidney to the right iliac fossa in Zhongshan Hospital of Sun Yat-sen University. The preparation of the donor kidney was uneventful, which included 1 vein and 1 artery. The recipient iliac fossa was exposed extraperitoneally. The graft's vein and artery was anastomosed end-to-side to the recipient's external iliac vein and artery, respectively. HLA typing showed three antigen mismatches. Direct microcytotoxic cross-match was negative. The cold ischemic time was approximately $6 \mathrm{~h}$, and the total warm ischemic time was $5 \mathrm{~min}$. Immunosuppression included antithymocyte globulin, prednisone, mycophenolate mofetil and tacrolimus. The patient showed immediate recovery of graft function and achieved a baseline serum creatinine level of $1.3 \mathrm{mg} / \mathrm{dL}$.

After one month, an asymptomatic rise in serum creatinine to $4.0 \mathrm{mg} / \mathrm{dL}$ was noted and the patient was readmitted to the hospital. Doppler ultrasonography showed a pseudoanerysm of the arterial graft anastomosis between renal transplant artery and external iliac artery. Computerized tomographic (CT) angiography also showed the anastomotic pseudoaneurysm $(4.0 \times 3.5 \mathrm{~cm})$ (Figure 1). Urine culture result was positive for Candida parapsilosis. After signing a consent form, she was 
taken to the operating room to undergo transplant nephrectomy. The patient received treatment with voriconazole for 3 months and hemodialysis.

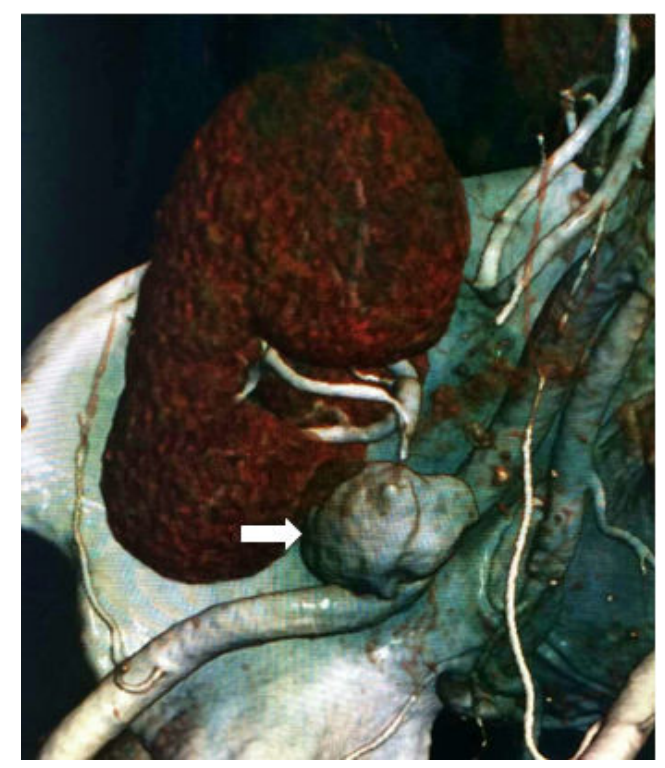

Figure 1. Computed tomographic angiography with 3-dimensional reconstruction showing a $4.0 \times 3.5 \mathrm{~cm}$ pseudoaneurysm (white arrow) at the junction between the donor renal artery and right external iliac artery.

\section{Renal recipient two}

Patient 2 was a 25-year-old man with end-stage renal disease caused by glomerulonephritis. Two years after starting hemodialysis, he underwent deceased-donor kidney transplantation of the left kidney to the right iliac fossa in the Second Xiangya Hospital of Central South University. The preparation of the donor kidney was uneventful, which included 1 vein and 2 arteries. The recipient iliac fossa was exposed extraperitoneally. An end-to-side anastomosis was done between the transplanted renal vein and the external iliac vein. The renal artery was anastomosed end-to-side to the external iliac artery because two renal arteries of similar diameters were using abdominal aorta patch. HLA typing showed two antigen mismatches. Direct microcytotoxic crossmatch was negative. The cold ischemic time was approximately $30 \mathrm{~h}$, and the total warm ischemic time was 5 min. Immunosuppression included antithymocyte globulin, prednisone, mycophenolate mofetil and tacrolimus. The recipient showed immediate recovery of graft function. The duplex ultrasonography routinely performed on day 14 showed that the graft was normal, and the patient was discharged from the hospital on the same day.

Sixteen days after transplantation, the patient developed abdominal pain that was very intense in the right hypochondrium, and was readmitted to the hospital with emergency. Physical examination found that blood was spurted from original drainage tube site. The patient received emergency surgery, in which rupture on the upper branch of the renal artery $2 \mathrm{~cm}$ from the arterial anastomosis site was found. Because of the difficulty to repair, the rupture was resected and anastomosis between the proximal and distal artery was performed. During the surgery, blood flow to the kidney was occluded for nearly $30 \mathrm{~min}$.

After operation, the patient developed a sudden decrease in diuresis. The cause for rupture was initially attributed to the surgical technique, but subsequent histopathologic examination showed the presence of Cryptococcus in the vascular bed of rupture (Figures 2A and 2B). Treatment with fluconazole (400 $\mathrm{mg} / \mathrm{d}$ ) was immediately started and continued for 6 months.

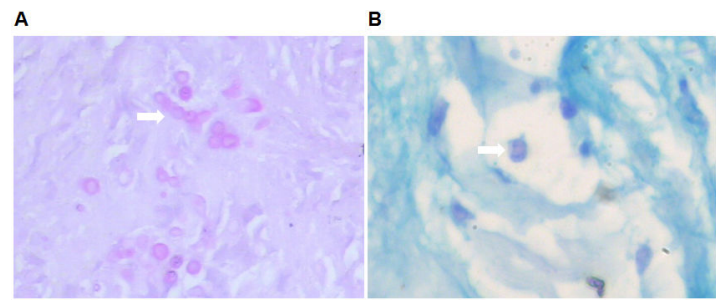

Figure 2. Histopathologic examination showing the presence of Cryptococcus in the vascular bed of rupture. (A) Purplish-red stained Cryptococcus (white arrow) in diseased renal artery (PAS stain; original magnification, 200X). (B) Blue stained Cryptococcus (white arrow) in diseased renal artery (Alcian blue stain; original magnification, 400X).

On day 21 after transplantation, Doppler ultrasound scan showed that there was little blood flow especially in the upper part of the kidney. Angiography confirmed that the upper transplanted renal artery was blocked. In addition, the patient presented with periallograft fluid collections on ultrasound, but test result of the accumulated fluid was negative. There was continuing drainage fluid until 3 months after secondary operation. Unfortunately, the graft function did not gain recovery and the patient was treated with hemodialysis.

\section{Liver recipient}

Liver recipient was a 43-year-old woman with end-stage liver disease caused by hepatitis B. The liver transplantation was performed in another organ transplant center. After transplantation, the patient did not have any infectious complication. Nine months after transplantation, the patient had an optimal graft functioning, in absence of infection.

\section{Discussion}

Mycotic arterial aneurysm or rupture following renal transplantation is an uncommon but dreaded complication, which may cause functional impairment and even loss of the graft. Growing evidences show that donor-derived fungal infections play important roles in the arterial aneurysm of renal transplant recipients [5]. Many factors such as immunosuppressive state, victims of near-drowning events, transplant-tourism practices and prolonged donor intensive care unit stay are considered to be associated with donorderived infections [4]. Among the fungi, candidiasis has been reported as the most common infection in renal transplant recipients [7]. The majority of donor-derived candidiasis is believed to be resulted from contamination of the preservation 
fluid, which can occur before or at the time of organ procurement. The clinical features of recipients may manifest as candidemia, abscess, aneurysm or renal artery rupture, among which vascular complications are the most severe consequences.

Rodrigues and colleagues [1] report six patients who have received grafts from donors with positive results in perfusion fluid testing for Candida. Among the six patients, four have had no evidence of fungal infection, but the other two have developed serious mycotic vascular complication resulting in nephrectomy. Mai and colleagues [8] have described the clinical course and outcomes in four renal transplantation recipients with Candida albicans due to contamination of preservation fluid. Two of the four patients have died from massive bleeding because of fungal arteritis, and the grafts in the other two cases have been removed. Therefore, these authors propose that nephrectomy should be compulsory in cases where preservation fluid is contaminated by Candida albicans. However, another study [9] shows favorable outcomes of renal transplantation in eight patients with Candida species contamination of preservation fluid. No clinical signs of fungal infections are observed in any patient and none of the patients has experienced vascular damage thanks to prophylactic antifungal treatment. Based on these cases, the researchers strongly suggest that graft removal should not be systematically proposed in such cases. Recently, Ministro and colleagues describe two patients with extrarenal pseudoaneurysm caused by Candida albicans after kidney transplantation. In both patients, the pseudoaneurysm diameter was larger than $2.5 \mathrm{~cm}$. They successfully preserve the kidney allograft with the treatment including excision of the infected tissue, arterial reconstruction with autologous tissue placed in previously unexposed tissue, and long-term antifungal therapy [10].

Most surgeons agree that recipients with mycotic aneurysm require transplant nephrectomy along with prolonged antifungal treatment [3,11], although a few cases of successfully preserved allograft function have been reported [12]. In our cases, one recipient is diagnosed with renal arterial aneurysm secondary to Candida parapsilosis, and transplant nephrectomy is performed in consideration of high mortality rates reported.

Cryptococcosis is considered to be the third most common mycotic infection in organ transplant recipients following candidiasis and aspergillosis [7]. Host factors, environmental factors, medications, and the type of transplant all play a role in the clinical presentation of Cryptococcosis [13]. Previous study indicates that cryptococcosis among transplant patients usually occurs more than one year after transplantation [14]. Although most transplant-associated cryptococcosis cases are resulted in from reactivation of recipient latent infection[6], there is a risk of transmission through donor organs or other tissues. A study [15] shows that cryptococcosis is confirmed in three recipients transmitted from a single donor with an undifferentiated neurologic condition. Among the three recipients, liver recipient and one kidney recipient have developed cryptococcemia and pneumonia, and the other kidney recipient has developed cryptococcemia and meningitis. Cryptococcal disease commonly leads to focal or mass lesions in central nervous system and lungs of patients with or without immune defects, and occasionally in skin, soft-tissue or osteoarticular $[14,16]$.

To our knowledge, the present report is the first report of Cryptococcus infection at graft sites. The infection occurs during the first 30 days after renal transplantation, leading to renal arterial rupture. According to guidelines of the American Society of Transplantation [4], Cryptococcus should be suspected of donor-derived transmission. In the present report, blood and urine culture results of the donor are negative before organ procurement, and mycotic infection in liver recipient is absent. However, the two renal recipients have developed different mycotic infections leading to severe vascular complications at the early stage of renal transplantation. Therefore, we conclude that the contamination of kidneys occurs during different periods of organ acquisition or preservation. In conclusion, vascular complications caused by fungal infections are very serious, and they can lead to graft loss and life-threatening situations. These infections can originate from either the donor or exogenous contamination during organ procurement or preservation. Early diagnosis and prompt management are critical for preventing fatal consequences.

\section{Acknowledgement}

We would like to thank the Second Xiangya Hospital of Central South University.

\section{References}

1. Rodrigues BF, Natario AS, Vizinho RS, Jorge CM, Weigert AL, Martinho A, Toscano C, Marques TT, Machado DS. Candida species contamination of preservation fluidoutcome of renal transplantation in 6 patients. Transplant Proc 2013; 45: 2215-2219.

2. Shoham S, Hinestrosa F, Moore J, O'Donnell S, Ruiz M, Light J. Invasive filamentous fungal infections associated with renal transplant tourism. Transpl Infect Dis 2010; 12: 371-374.

3. Albano L, Bretagne S, Mamzer-Bruneel MF, Kacso I, Desnos-Ollivier M, Guerrini P, Le Luong T, Cassuto E, Dromer F, Lortholary O. Evidence that graft-site candidiasis after kidney transplantation is acquired during organ recovery: a multicenter study in France. Clin Infect Dis 2009; 48: 194-202.

4. Singh N, Huprikar S, Burdette SD, Morris MI, Blair JE, Wheat LJ. Donor-derived fungal infections in organ transplant recipients: guidelines of the American Society of Transplantation, infectious diseases community of practice. Am J Transplant 2012; 12: 2414-2428.

5. Gomez CA, Singh N. Donor-derived filamentous fungal infections in solid organ transplant recipients. Curr Opin Infect Dis 2013; 26: 309-316. 
6. Saha DC, Goldman DL, Shao X, Casadevall A, Husain S, Limaye AP, Lyon M, Somani J, Pursell K, Pruett TL, Singh N. Serologic evidence for reactivation of cryptococcosis in solid-organ transplant recipients. Clin Vaccine Immunol 2007; 14: 1550-1554.

7. Pappas PG, Alexander BD, Andes DR, Hadley S, Kauffman CA, Freifeld A, Anaissie EJ, Brumble LM, Herwaldt L, Ito J, Kontoyiannis DP, Lyon GM, Marr KA, Morrison VA, Park BJ, Patterson TF, Perl TM, Oster RA, Schuster MG, Walker R, Walsh TJ, Wannemuehler KA, Chiller TM. Invasive fungal infections among organ transplant recipients: results of the Transplant-Associated Infection Surveillance Network (TRANSNET). Clin Infect Dis 2010; 50: 1101-1111.

8. Mai H, Champion L, Ouali N, Hertig A, Peraldi MN, Glotz D, Rondeau E, Costa MA, Snanoudj R, Benoit G, Charpentier B, Durrbach A. Candida albicans arteritis transmitted by conservative liquid after renal transplantation: a report of four cases and review of the literature. Transplantation 2006; 82: 1163-1167.

9. Matignon M, Botterel F, Audard V, Dunogue B, Dahan K, Lang P, Bretagne S, Grimbert P. Outcome of renal transplantation in eight patients with Candida sp. contamination of preservation fluid. Am J Transplant 2008; 8: 697-700.

10. Ministro A, Ferreira T, Batista L, Santana A, Alves N, Guerra J, Fernandes E Fernandes J. Mycotic Pseudoaneurysm After Kidney Transplantation: Two Case Reports. Transplant Proc 2017; 49: 906-912.

11. Patrono D, Verhelst R, Buemi A, Darius T, Godefroid N, Mourad M. Presentation and management of mycotic pseudoaneurysm after kidney transplantation. Transpl Infect Dis 2015; 17: 129-136.
12. Che H, Men C, Yang M, Zhang J, Chen P, Yong J. Endovascular repair of a transplant renal artery anastomotic pseudoaneurysm using the snorkel technique. J Vasc Surg 2014; 60: 1052-1055.

13. Henao-Martínez AF, Beckham JD. Cryptococcosis in solid organ transplant recipients. See comment in PubMed Commons below Curr Opin Infect Dis 2015; 28: 300-307.

14. Singh N, Alexander BD, Lortholary O, Dromer F, Gupta KL, John GT, del Busto R, Klintmalm GB, Somani J, Lyon GM, Pursell K, Stosor V, Munoz P, Limaye AP, Kalil AC, Pruett TL, Garcia-Diaz J, Humar A, Houston S, House AA, Wray D, Orloff S, Dowdy LA, Fisher RA, Heitman J, Wagener MM, Husain S. Cryptococcus neoformans in organ transplant recipients: impact of calcineurin-inhibitor agents on mortality. J Infect Dis 2007; 195: 756-764.

15. Baddley JW, Schain DC, Gupte AA, Lodhi SA, Kayler LK, Frade JP, Lockhart SR, Chiller T, Bynon JS, Bower WA. Transmission of Cryptococcus neoformans by Organ Transplantation. Clin Infect Dis 2011; 52: e94-98.

16. Yoneda T, Itami Y, Hirayama A, Saka T, Yoshida K, Fujimoto K. Cryptococcal necrotizing fasciitis in a patient after renal transplantation-a case report. Transplant Proc 2014; 46: 620-622.

\section{*Correspondence to}

Longkai Peng

Center of Organ Transplantation

The Second Xiangya Hospital of Central South University

PR China 